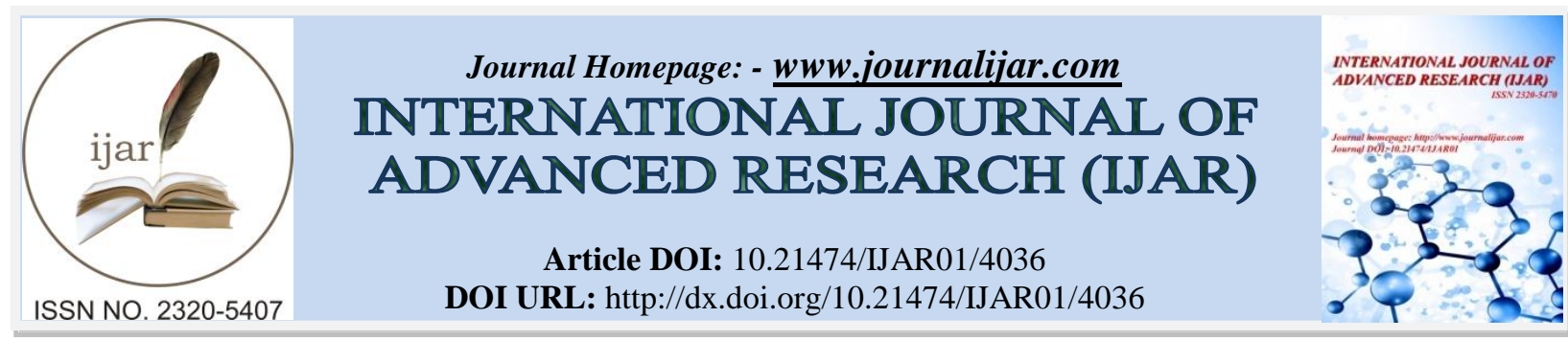

RESEARCH ARTICLE

\title{
A DOSIMETRIC STUDY OF CONFORMAL RADIOTHERAPY PLAN WITH WEDGE AND FIELD-IN- FIELD-FORWARD-PLANNED RADIOTHERAPY IN LEFT SIDED BREAST CANCER.
}

\author{
"Mohamed abdelgawad ${ }^{1}$, Amira elwan ${ }^{1}$ and Emad mostafa ${ }^{2}$. \\ 1. Clinical oncology department, Faculty of Medicine, Zagazig University, Egypt. \\ 2. Medical physicist, Clinical oncology department, Faculty of Medicine, Zagazig University , Egypt.
}

\section{Manuscript Info}

Manuscript History

Received: 25 February 2017

Final Accepted: 16 March 2017

Published: April 2017

Key words:-

Dosimetric, ,Breast cancer, conformal

$\mathrm{RT}$, field in field plan

\begin{abstract}
Purpose: To perform a dosimetric comparison of field in field forward planned radiotherapy (FiF-FP) and (3D-CRT) with wedge which are used in whole breast irradiation.

Method: In thirty patients, two techniques per patient were done (3DCRT )with wedge and (FiF-FP).A comparison of treatment plans was done, using dose volume histograms for (PTV) and organs at risk. Volumes: V95, V105, D mean, D max, D min V20, V30 and V5 were chosen for the comparison.

Results: The 2 plans, revealed a comparable good dose coverage, V95 $>95 \%$ of PTV. V105\% of doses were observed in less than $10 \%$ of the (PTV). D max. $<110 \%$ of prescribed doses in FiF-FP and $>110 \%$ for 3D-CRT. D mean of PTVs were $101.1 \%$ for 3D-CRT versus $100.1 \%$ for FiF-FP ,all values were less than the tolerance doses of OARs, the two plans were equivalent in sparing (OARs), V20 Gy and V30 Gy for heart and ipsilateral lung in the 3D-CRT had an edge over FiF-FP. $\mathrm{D}$ max of the heart and ipsilateral lung were higher in 3D-CRT than FiF-FP , D max was lower in 3D-CRT than FiF-FP for contralateral breast. (MUs ) needed to deliver 3D-CRT with wedge plan were more than the values needed to deliver FiF-FP.

Conclusion: FiF-FP had better dose homogeneity in (PTV) than (3DCRT) with wedge and with less dose to OARs. (MUs) were less in (FiF-FP) with less time on the machine and less machine consumption. This technique lead to good expected breast irradiation outcome and encourages breast conservative surgery in developing countries

Copy Right, IJAR, 2017,. All rights reserved.
\end{abstract}

\section{Introduction:-}

Radiotherapy Postoperatively is a standard in combined treatment of breast cancer. Conventional radiation is relied on tangential portals. Most frequently prescribed dose is fifty gray in twenty five fractions in five weeks (1). Ordinary arranging of dose distribution on the basis of two tangential wedged fields, regularly comes out suboptimal doses to the target volume; particular part of the target volume receives less than ninety five percent of the dose and another part receives more than one hundred and seven percent of the dose. The distribution factor negatively affects the cosmetic effect as well as the treatment outcome (2-4). The radiation techniques of breast cancer treatment differ at different establishments, radiation dose issue conveyance to the post mastectomy chest wall or to the breast after conservation stays difficult. In the conventional breast radiotherapy strategy, the beam arrangement composed of two tangential glancing portals (5), with acceptable coverage of the target, while decrease the dose to neighboring 
basic structures (i.e. lung, heart, and normal breast). dynamic or Physical wedges are usually added to these tangential beams so as for changes compensation in external contours and to improve the entire breast dose uniformity (6).

Many researchers have explained the different strategies including intensity modulated radiotherapy, three noncoplanar conformal fields, field-in-field technique for dosimetric improvements, benefits and sparing of organs at risk, (8). The field in field technique helps to qualify dose homogeneity to the planning target volume (PTV) while decreasing the absorbed dose in irradiated organs near the PTV(7).

\section{Aim of work:-}

Our aim to perform a comparable study of field in field radiotherapy and three dimensional conformal radiotherapy with wedge used in whole breast irradiation from dosimetric point of view.

\section{Methodology:-}

We included thirty patients with left breast cancer who underwent conservative surgery from May 2014 and August 2016 at Clinical Oncology Department, Zagazig University, Egypt. Patients received a dose as fifty gray to the whole breast in twenty five fractions, the tumor bed was covered by a boost dose after whole breast irradiation , 6-15MV photon beam was probably selected according to the plan for better dose distributions. Enrollment of patients with left breast cancer to evaluate doses received to the heart. The planning system was Precise treatment planning station. Two techniques were used for the treatment planning ,three dimensional conformal radiotherapy (3D-CRT) with wedge and Field-in-Field-Forward-planned (FiF-FP). Tangential plan technique consisting of medial and lateral fields to treat the breast.

\section{Procedures:-}

Patients were positioned supine comfortably and the arms above the head, immobilized on the breast board . Computed tomography (CT ) stimulation was done for each case and it was done from the angle of the mandible to $5 \mathrm{~cm}$ below the breast, of $3 \mathrm{~mm}$ thickness.

The target volumes and organs at risk like the heart, the ipsilateral lung, and the contra-lateral breast, were delineated. The intact breast PTVs were delineated 3-5 mm under the skin surface because of build-up region from the PTVs. The borders of fields were defined by radio-opaque wires at simulation step . The field borders were defined as :- medially, midline ,superiorly, lower border of clavicle, $2 \mathrm{~cm}$ around the defined breast parenchyma for inferior and lateral border.

\section{Comparison of Treatment Techniques:-}

A comparison between two techniques was created to evaluate the dosimetric parameters:-

1) Conformal Plan with wedge (3D-CRT) : we used two parallel opposing tangential beams to cover the breast tissue, to diminish organs at risk dose(i.e. contralateral breast, ipsilateral lung and heart). Wedges were then added to medial and lateral tangential beams for better dose homogeneity to the (PTV) in trials to decrease dose volumes to the heart and lung as permissible .

2) Field in Field Forward planned (FiF-FP):

In this technique, we used two open tangential fields according to the geometry defined during simulation for better to the breast volume dose distribution. Firstly equal weights were created to the two open tangs .We calculated the better dose distribution. Subfields from 3 to 4 fields were manually created by multi-leaf Collimator (MLC) to improve dose homogeneity and to decrease overdose in the (PTV). Subfields were created by the copy of the tangential fields, and pasted it as an open field. In BEV, with the help of MLC, we shielded the volume which received more than hundred of the prescribed dose. subfields were created by manually fitted MLC's to "hot" areas. Opened beam area receives query eighty percent of the dose and the subfields contribute query twenty percent Finally, the main field and subfields were combined in one portal.

\section{Plans Evaluation:-}

The dose volume histograms (DVHs) were done for (PTVs) and Organs at Risk (OARs).

The dosimetry parameters that were used are:

1) V95 is the volume of (PTV) received $95 \%$ of the prescribed dose.

2) V105 is the volume of (PTV) receives $105 \%$ of the prescribed dose.

3)D. mean and D. max are the mean and maximum dose of (PTV) and (OARs). 
4) D. min is the minimum dose received to (PTV).

5) V20 and V30 are the volume of the heart and the ipsilateral lung receive $20 \%$ and $30 \%$ of the prescribed dose.

6) V5 is the volume of the contralateral breast receives $5 \%$ of the prescribed dose.

7) Quality of coverage ${ }_{\mathrm{RTOG}}=$ Minimal isodose surrounding the target / Reference isodose).

8) Homogeneity index ${ }_{R T O G}=$ Maximum isodose in the target / Reference isodose).

\section{Results:-}

Dosimetric characteristics of (PTV): We observed that the 2 plans (3D-CRT) and ( FiF-FP) revealed a comparable good dose coverage. V95 was greater than $95 \%$ of (PTV) with statistical non significance $(\mathrm{p}=.671)$. V105\% of doses were observed in less than $10 \%$ of the PTV with statistical significance $(\mathrm{p}=.000)$. The mean doses of (PTV )were 101.1 for (3D-CRT ) versus 100.1 for (FiF-FP ) with statistical significance $(\mathrm{p}=.041)$ (Table 1).

Homogeneity index of PTVs were of statistical significance for 3D-CRT versus FiF-FP respectively, (1.11 \pm 0.01 vs $1.08 \pm 0.01)(\mathrm{p}=.001)$, while quality of PTVs coverage were of statistical non significance $0.8 \pm 0.03 \mathrm{vs} 0.8 \pm 0.06(\mathrm{p}$ $=.565)$ (Table 1).

Dosimetric characteristics of Organs At Risks (OARs): We observed that all the values were less than the tolerance doses of OARs. Also, the two plans were equivalent in sparing risk organs. The differences between planning techniques shown statistical significance in mean doses to the contralateral breast, heart and ipsilateral lung with a small decrease of the mean doses to OARs were present in the FiF-FP in comparison to 3D-CRT(Table 2).. The doses of volume data for OARs( ipsilateral lung and heart ) in terms of V20 Gy and V30 Gy for (3D-CRT )treatment plans had an edge over( FiF-FP )plans with a statistical significance(Table 2).

Maximum doses received were higher for 3D-CRT treatment plans than FiF-FP plans with a statistical significance as in D-max of the heart and non-statistical significance in D-max of the ipsilateral lung (Table 2).

Table1:- Comparison of the dosimetric characteristics of PTV.

\begin{tabular}{|l|l|l|l|}
\hline \multirow{2}{*}{ Dose Parameter } & 3D-CRT with wedge & FiF-FP & P value \\
\cline { 2 - 3 } & Mean $\pm \mathrm{SD}(\%)$ & Mean $\pm \mathrm{SD}(\%)$ & .041 \\
\hline D mean & $101.1 \pm 1$ & $100.1 \pm 2.3$ & $.000^{*}$ \\
\hline D maximum & $111 \pm 1.43$ & $108.8 \pm 1.45$ & .558 \\
\hline V95 minimum & $80.3 \pm 3.2$ & $80.8 \pm 6.8$ & .671 \\
\hline V105 & $95.4 \pm 2.5$ & $95.2 \pm 1.7$ & $.000^{*}$ \\
\hline Homogeneity index & $6.7 \pm 1.6$ & $4.6 \pm 1.5$ & .001 \\
\hline Quality of coverage & $1.11 \pm 0.01$ & $1.08 \pm 0.01$ & .565 \\
\hline
\end{tabular}

PTV,planning target voulum , 3D-CRT ,3D-Conformal Plan with wedge,

FiF-FP,Field-in-Field-Forward-planned -* Highly significant.

Table2:- Comparison of the dosimetric characteristics of OARs

\begin{tabular}{|c|c|c|c|c|}
\hline \multirow{2}{*}{\multicolumn{2}{|c|}{ Dose parameter }} & \multirow{2}{*}{$\begin{array}{l}\text { 3D-CRT with wedge } \\
\text { Mean } \pm \mathrm{SD}(\%)\end{array}$} & \multirow{2}{*}{$\begin{array}{l}\text { FiF-FP } \\
\text { Mean } \pm \mathrm{SD}(\%)\end{array}$} & \multirow{2}{*}{$\begin{array}{l}\mathbf{P} \\
\text { value }\end{array}$} \\
\hline & & & & \\
\hline \multirow{4}{*}{$\begin{array}{l}\text { Ipsilateral } \\
\text { lung }\end{array}$} & D mean & $16.7 \pm 4.6$ & $16.2 \pm 3.5$ & $.000 *$ \\
\hline & D maximum & $99.4 \pm 1.1$ & $96.5 \pm 1.6$ & .587 \\
\hline & V20 & $5.9 \pm 2.5$ & $5.1 \pm 2$ & $.000 *$ \\
\hline & V30 & $4.06 \pm 1.9$ & $3.4 \pm 1.7$ & $.000 *$ \\
\hline \multirow[t]{4}{*}{ Heart } & D mean & $12.7 \pm 5.2$ & $7.8 \pm 1.7$ & .001 \\
\hline & $\mathrm{D}$ maximum & $89.3 \pm 1.7$ & $85.2 \pm 4.1$ & $.000 *$ \\
\hline & V20 & $16.5 \pm 4.3$ & $15.4 \pm 5.2$ & .001 \\
\hline & V30 & $16.06 \pm 5.5$ & $14.5 \pm 4.6$ & $.000 *$ \\
\hline \multirow{3}{*}{$\begin{array}{l}\text { Contralateral } \\
\text { breast }\end{array}$} & D mean & $2.5 \pm .08$ & $1.1 \pm .014$ & $.000 *$ \\
\hline & D maximum & $23.6 \pm 2.01$ & $24.06 \pm 3.4$ & .337 \\
\hline & V5 & $1.08 \pm 0.44$ & $0.8 \pm 0.3$ & $.000 *$ \\
\hline
\end{tabular}


Table 3:- Comparison of the monitor units(MUs) used for both technique .

\begin{tabular}{|l|l|l|l|}
\hline Dose Parameter & 3D-CRT with wedge & FiF-FP & P \\
\cline { 2 - 4 } & Mean $\pm \operatorname{SD}(\%)$ & Mean $\pm \operatorname{SD}(\%)$ & $.000^{*}$ \\
\hline Medial tangs & $162.16 \pm 5$ & $113 \pm 2.2$ & $.000^{*}$ \\
\hline Lateral tangs & $156.5 \pm 5.4$ & $107 \pm 1.6$ & $.000^{*}$ \\
\hline Total MU & $318.6 \pm 10.3$ & $220 \pm 2.9$ & \\
\hline
\end{tabular}

Table 3: Shows the monitor Units (MUs) for the 2 plans. The mean of total MUs needed to deliver 3D-CRT with wedge plan was $(318.6 \pm 10.3)$ which was more than the values needed to deliver FiF-FP(220 \pm 2.9$)$ with statistical significance $(\mathrm{p}=.000)$

\section{Discussion:-}

Conservative surgery and postoperative RT is an important treatment for early stage breast cancer.Various 3D CT breast radiotherapy techniques facilitate a homogenous dose distribution in PTV while sparing OARs. The tangential field technique with wedge filters, which is used to optimize the dose distribution, have been shown to provide an excellent local control with rare long term complications $(10,11)$. The main drawbacks of wedge technique are as the angle increases, the scatter component from the wedge also increases resulting in increased patients undesired dose (12-14). Also with treatment plans performed with tangential fields, as the wedge angle used to decrease the extra dose at the top of the breast results in the medial and lateral beam entries dose increase (8).Many investigators showed that use of the field in field plan improve dose distribution during whole breast irradiation $(8,9,15-18)$. The multileaf collimator is used instead of wedges for field in field technique. The use of multileaf collimator decreasing scattered doses to OARs when compared to conventional tangential field technique with wedges (19-20). Moreover, some hot inhomgenities may stay after the use of wedge because of extreme tissue geometric extremes, this can be avoided by adopting the field in field technique (12).

In the present study a comparison of field in field radiotherapy and conformal radiotherapy with wedge which are usually used in whole breast irradiation in left-side breast cancer patients and found that field in field technique was superior to CRT technique with wedge in terms of dose homogeneity in PTV and absorbed dose in OARs.

In our study, V105 of PTV, V20, V30 for the heart and lung, V5 for the contralateral breast in the field in field arm were less with statistical significance. Also, V95 of PTVs in the field in field arm were decreased with nonsignificance.

G. Yavas et al, reported that, the FIF technique significantly reduced the V5, V10, V20, V30 and V40 values of the contralateral breast while heart volumes receiving 2, 30 and $40 \mathrm{~Gy}$ were decreased significantly. Similarly, V2, V10, V20 and V30 and V40 values for the ipsilateral lung were significantly reduced with FIF technique when compared to CRT technique(7).

In our study, there was decrease of the mean dose to OARs in the FiF-FP than 3D-CRT plan : contralateral breast $(1.1 \pm .014$ versus $2.5 \pm .08)$,heart : $(7.8 \pm 1.7$ versus $12.7 \pm 5.2)$ and ipsilateral lung :(16.2 \pm 3.5 versus $16.7 \pm 4.6)$ respectively.

Zakiya Salem Al-Rahbi et al, reported that the mean doses to ipsilateral lung, contralateral breast and heart OARs (V20 ipsi.lung, contralateral breast, V30 Heart,) are the least with IP-intensity modulated radiotherapy than other techniques of conformal radiotherapy CRT, FiF-IMRT , and electronic tissue compensation methods( E-COM)(6). Many studies resulted in more risk incidence of second cancer of contralateral breast irradiation dose (21).

Our results revealed that the monitor units needed to deliver 3D-CRT with wedge plan $(318.6 \pm 10.3)$ were more than the values needed to deliver FiF-FP(220 2.9$)$ with statistically significance ( $\mathrm{p}=.000)$, Zakiya Salem AlRahbi et al, reported that the average monitor units used in inverse plan of intense modulated radiotherapy (437) were about twice the value needed to deliver 3Dimensional conformal radiotherapy (P) (278), 3D-CRT (D)(235) and Field in field IMRT(227) (6), Guang-Hua Jin et al, reported that the average monitor units of VMAT plan (363.7 \pm 45.3$)$ were significantly smaller $(\mathrm{P}<0.05)$ than that of 7-IMRT $(513.4 \pm 83.3 \mathrm{MU})$.VMAT technique was better in the irradiation MUs in comparison to 7-IMRT. Also, VMAT plan had advantages in reducing the volume of high dose with increased volume of lower dose(22)., G. Yavas et al, reported that the monitor units required for the FIF 
technique were lower when compared to CRT technique(7). In field in field strategy, the monitor units were adjusted among the sub-fields and even an increase in the number of sub-fields wouldn't have much change in the MUs. This is the largest value of using FIF strategy in irradiation (12).

\section{Conclusion:-}

Field in-field forward planned technique had better PTV dose homogeneity than conformal Radiotherapy with wedge and with less doses to OARs. The used monitor Units (MUs) were less in Field-in-Field-Forward-planned with less time on the machine and less machine consumption. This technique improves breast irradiation outcome and encourages breast conservative surgery in developing countries.

\section{Reference:-}

1. Morganti AG, Cilla S, Valentini V, Digesu C, et al. Phase I-II studies on accelerated IMRT in breast carcinoma: techni- cal comparison and acute toxic- ity in 332 patients. Radiother Oncol. 2009;90(1):86-92. PMID: 19010560 DOI:10.1016/j.radonc.2008.10.017.

2. Gray JR, McCormick B, Cox L, Yaha- lom J. Primary breast irradiation in large-breasted or heavy women: anal- ysis of cosmetic outcome. Int J Radiat Oncol Biol Phys. 1991;21(2):347-54. PMID:2061111.

3. Neal AJ, Mayles WP, Yarnold JR. Invited review: tangential breast irradiation--rationale and methods for improving dosimetry. Br J Radiol. 1994;67(804):1149-54. PMID:7874411 DOI:10.1259/0007-1285-67-8041149.

4. Neal AJ, Torr M, Helyer S, Yarnold JR. Correlation of breast dose het- erogeneity with breast size using 3D CT planning and dose-volume histograms. Radiother Oncol.1995;34(3):210-8. PMID:7631027.

5. A. Recht, "Breast Cancer: Stages T1 and T2," In: L. L. Gunderson and J. E. Tepper, Eds., 2nd Edition, Clinical Radiation Oncology, Churchill Livingstone, Elsevier Pub- lications, 2007, pp. 1475-1495.

6. Zakiya Salem Al-Rahbi, Ramamoorthy Ravichandran, Johnson Pichy Binukumar, et al: A Dosimetric Comparison of Radiotherapy Techniques in the Treatment of Carcinoma of Breast; Journal of Cancer Therapy, 2013, 4, 10-17.

7. G. Yavas, C. Yavas, H. Acar:Dosimetric comparison of whole breast radiotherapy using field in field and conformal radiotherapy techniques in early stage breast cancer; Iran. J. Radiat. Res., 2012; 10(3-4): 131-138.

8. Ercan $\mathrm{T}$, İgdem $\mathrm{S}$, Alco $\mathrm{G}$, Zengin $\mathrm{F}$, et al: Dosimetric comparison of field in field intensity-modulated radiotherapy technique with conformal radiotherapy techniques in breast cancer. Jpn J Radiol, 2010; 28:283289. PMID:20512546 DOI:10.1007/s11604-010-0423-3.

9. Sasaoka M and Futami T: Dosimetric evaluation of whole breast radiotherapy using field-in-field technique in early-stage breast cancer. Int J Clin Oncol, 2011;16:250-256. PMID:21229283 DOI:10.1007/s10147-010-01751.

10. Romestaing $\mathrm{P}$, Lehingue $\mathrm{Y}$, Carrie $\mathrm{C}$, et al. Role of a $10 \mathrm{~Gy}$ boost in the conservative treatment of early breast cancer: results of a randomized clinical trial in Lyon, France. J Clin Oncol, 1997; 15: 963-968. PMID: 9060534.

11. Bartelink H, Horiot JC, Poortmans PM, et al.: Impact of a higher radiation dose on local control and survival in breast-conserving therapy of early breast cancer: 10-year results of the randomized boost versus no boostEORTC 22881-10882 trial. J Clin Oncol, 2007; 25: 3259-3265. PMID:17577015 DOI:10.1200/JCO.2007.11.4991.

12. Prabhakar R, Julka PK, Rath GK Can field-in-field technique replace wedge filter in radiotherapy treatment planning: a comparative analysis in various treatmentsites. Australas Phys Eng Sci Med, 2008 ; 31: 317-324. PMID:19239058.

13. Prabhakar R, Julka PK, Malik M, et al :Comparison of contralateral breast dose for various tangential field techniques in clinical radiotherapy. Technol Cancer Res Treat, 2007; 6: 135-8. PMID:17375976.

14. Stasi M, Moro G, Ramella S, Bertone, A, et al :Factors affecting the contralateral dose for the non-treated breast in irradiation following quadrantectomy. Radiol Med, 1997; 93: 596-9. PMID:9280945.

15. Zackrisson B, Arevarn M, Karlsson M :Optimized MLCbeam arrangement for tangential breast irradiation. Radiother Oncol, 2000;54:209-12. PMID:10738078.

16. Richmond ND, Turner RN, Dawes PJDK et al.: Evaluation of the dosimetric consequences of adding a single asymmetric or MLC shaped field to a tangential breast radiotherapy technique. Radiat Oncol, 2003;67: 165170.

17. Donovan EM, Johnson U, Shentall G et al.: Evaluation of compensation in breast radiotherapy: a planning study using multiple static fields. Int J Radiat Biol Phys, 2000;46:671-679 PMID:10701747. 
18. Lee JW, Hong S, Choi KS et al. :Performance evaluation of field-in-field technique for tangential breast irradiation. Jpn J Clin Oncol, ;2008; ; 38:158-163. PMID:18216025 DOI:10.1093/jjco/hym167.

19. Bhatnagar AK, Brandner E, Sonnik D et al. Intensity modulated radiation therapy (IMRT) reduced the dose to the contralateral breast when compared to the conventional tangential fields for primary breast irradiation. Breast Cancer Res Treat, 2006; 96:41-46.

20. Woo TC, Pignol JP, Rakovitch E et al. Body irradiation exposure in breast cancer radiotherapy: impact of breast IMRT and virtual wedge compensationtechniques. Int $\mathrm{J}$ Radiat Oncol Biol Phys, 2006; 1:52-58. PMID:16457966 DOI:10.1016/j.ijrobp.2005.11.023.

21. Hong L, Hunt M, Chui C, et al: Intensity-modulated tangential beam irradiation of the intact breast. Int J Radiat Oncol Biol Phys 1999, 44:1155-1164. PMID:10421550

22. Guang-Hua Jin, Li-Xin Chen, Xiao-Wu Deng et al: A comparative dosimetric study for treating left-sided breast cancer for small breast size using five different radiotherapy techniques: conventional tangential field, filed-in-filed,Tangential-IMRT, Multi-beam IMRT and VMAT, Jin et al. Radiation Oncology 2013, 8:89. 